\title{
NOTE ON SEQUENCES OF MAYER-VIETORIS TYPE
}

\author{
ELDON DYER AND JOSEPH ROITBERG
}

\begin{abstract}
In this largely expository note, we reexamine the construction of the homotopical Mayer-Vietoris sequence associated to a homotopy pullback. We show that in this situation, the Mayer-Vietoris sequence may be realized simply as the homotopy sequence of a suitable fibration. The usual approaches to constructing the Mayer-Vietoris sequence involve some auxiliary algebraic result, such as the Barratt-Whitehead lemma; the present approach avoids any such considerations. An additional beneficial feature of our approach is the attention paid to the bottom end of the Mayer-Vietoris sequence. Thus we are led to a cleaner proof of Proposition II.7.11 of [HMR]; moreover, we show that the converse of this latter result is also true.

The homological Mayer-Vietoris sequence associated to a homotopy pushout may be established in a very similar manner, as we point out at the end of the paper.
\end{abstract}

1. We work throughout in the category $\mathcal{T}$ of pointed (not necessarily path-connected) topological spaces. Given $B \in \mathcal{T}$, we denote by $B$ the space of all (not necessarily pointed) paths in $B$ and by $\varepsilon: B \rightarrow B \times B$ the "endpoints map" defined by $\varepsilon(\pi)=(\pi(0), \pi(1))$. We then have

Proposition. The map $\varepsilon: B \rightarrow B \times B$ is a (Hurewicz) fibration with fiber $\Omega B$, the loop space of $B$. The connecting map $\partial: \Omega B \times \Omega B=\Omega(B \times B) \rightarrow \Omega B$ associated to this fibration is given by $\partial(\lambda, \mu)=\lambda \cdot \mu^{-1}$, that is, $\partial=m \circ(1 \times(-1))$, where $m$ is the standard multiplication on $\Omega B$.

The proof is standard. Nevertheless, the second assertion of the Proposition is perhaps not as well known as it deserves to be; it forms the basis for what follows.

Consider now a diagram

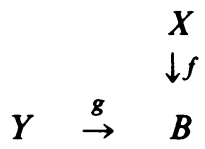

in $\mathscr{T}$. By its very definition, the homotopy pullback $Z$ of (1.1) may be viewed as the total space of the fibration induced from the fibration of the Proposition by means of the map $f \times g: X \times Y \rightarrow B \times B$. Thus we have a commutative diagram

Received by the editors December 28, 1979.

AMS (MOS) subject classifications (1970). Primary 55R05, 55P05.

(C) 1980 American Mathematical Society $0002-9939 / 80 / 0000-0628 / \$ 01.75$ 


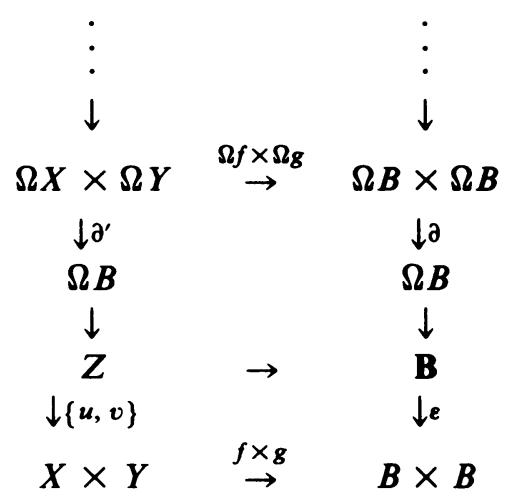

Note that (1.1) may be completed to a homotopy-commutative diagram

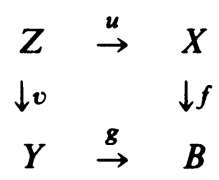

and that if either $f$ or $g$ is a fibration, then $Z$ is homotopy equivalent to the strict pullback of (1.1); see [HMR].

Passing to the homotopy sequence of the fibration

$$
\Omega B \rightarrow Z \stackrel{\{u, v\}}{\rightarrow} X \times Y,
$$

taking into account the commutativity of the upper square in (1.2) together with the Proposition, we obtain

THEOREM. There is a long exact sequence

$$
\begin{aligned}
\cdots & \rightarrow \pi_{n+1} B \rightarrow \pi_{n} Z \stackrel{\left\{u_{*}, v_{*}\right\}}{\rightarrow} \pi_{n} X \oplus \pi_{n} Y \stackrel{f_{*}-g_{*}}{\rightarrow} \pi_{n} B \rightarrow \cdots \\
& \rightarrow \pi_{2} B \rightarrow \pi_{1} Z \stackrel{\text { (u*, }}{\rightarrow} \pi_{1} X \times \pi_{1} Y \stackrel{f_{*} \cdot g_{*}^{-1}}{\rightarrow} \pi_{1} B \rightarrow \pi_{0} Z \rightarrow \pi_{0}(X \times Y) .
\end{aligned}
$$

This may be taken to be the definition of the Mayer-Vietoris sequence associated to the homotopy pullback (1.3). It is a simple matter to check that this sequence agrees with the usual Mayer-Vietoris sequence, as derived, for example, in [EH].

By studying the bottom end of our Mayer-Vietoris sequence, the following consequence is deduced.

Corollary. If, in (1.3), $X$ and $Y$ are path-connected and every $\gamma \in \pi_{1} B$ is expressible in the form $\gamma=f_{*} \alpha \cdot g_{*} \beta$ for some $\alpha \in \pi_{1} X, \beta \in \pi_{1} Y$, then $Z$ is path-connected.

Conversely, if $Z$ is path-connected, then every $\gamma \in \pi_{1} B$ is expressible in the form $\gamma=f_{*} \alpha \cdot g_{*} \beta$ for some $\alpha \in \pi_{1} X, \beta \in \pi_{1} Y$; if, in addition, $B$ is path-connected, then $X$ and $Y$ are also path-connected.

The first assertion of the Corollary is Proposition II.7.11 of [HMR]. (The latter unnecessarily stipulates that $B$ be path-connected.) The converse does not seem to have been previously noted, at least in print. 
2. It is quite straightforward to dualize the constructions of $\$ 1$ so as to realize the Mayer-Vietoris sequence associated to a homotopy pushout as the homology sequence of a suitable cofibration. The dual of the "universal example" fibration $\varepsilon$ : $\mathbf{B} \rightarrow B \times B$ is the "universal example" cofibration $i: A \vee A \rightarrow \operatorname{cyl} A, \operatorname{cyl} A$ denotes the reduced cylinder over $A$ and $i$ the natural inclusion. ${ }^{1}$ The cofiber of $i$ is $\Sigma A$, the reduced suspension of $A$ and the associated connecting map $\delta: \Sigma A \rightarrow$ $\Sigma(A \vee A)=\Sigma A \vee \Sigma A$ is given by composing the standard comultiplication $c$ : $\Sigma A \rightarrow \Sigma A \vee \Sigma A$ with the map $1 \vee(-1): \Sigma A \vee \Sigma A \rightarrow \Sigma A \vee \Sigma A$, as is readily seen. We may safely leave further details of the dualization of $\S 1$ to the reader. (Observe that the Corollary of $\$ 1$ has no analogue here.)

\section{BIBLIOGRAPHY}

[EH] B. Eckmann and P. Hilton, Unions and intersections in homotopy theory, Comment. Math. Helv. 38 (1964), 293-307.

[HMR] P. Hilton, G. Mislin and J. Roitberg, Localization of nilpotent groups and spaces, North-Holland Math. Studies, No. 15, North-Holland, Amsterdam, 1975.

Department of Mathematics, Graduate School and University Center (CUNY), New York, New YoRK 10036

Department of Mathematics, hunter College and Graduate School (CUNY), New York, NEW YORK 10021

'Actually, in order to insure that $i$ is a cofibration, it should be assumed that the basepoint of $A$ is cofibered in $A$. Alternatively, we could work in the category of unpointed topological spaces and replace $i$ by the natural inclusion of the disjoint union $A+A$ into the cylinder $A \times I$. 